\title{
A Deep Learning Framework in FCC Process Control
}

\author{
Q Y YAN ${ }^{\mathrm{a}}, \mathrm{X} \mathrm{LIN}^{\mathrm{b}, \uparrow}, \mathrm{Z}_{\mathrm{Q}} \mathrm{Q} \mathrm{QIN}^{\mathrm{b}}, \mathrm{G} \mathrm{L} \mathrm{LUO}^{\mathrm{c}}, \mathrm{D}_{\mathrm{Q}} \mathrm{Q} \mathrm{WANG}^{\mathrm{d}}$ and X S XIAO${ }^{\mathrm{e}, 1}$ \\ ${ }^{a}$ College of Transportation Engineering, Dalian Maritime University, Dalian 116085, \\ China \\ ${ }^{\mathrm{b}}$ School of Management, Zhejiang University, Hangzhou, 310058, China \\ c School of Pharmacy, Shenyang Pharmacy University, Benxi 117004, China \\ ${ }^{\mathrm{d}}$ College of Control Science and Engineering, Zhejiang University, Hangzhou 310027, \\ China \\ e Bigdata Technology Institute, Guizhou Light Industry Polytechnic College, Guiyang \\ 550025, China
}

\begin{abstract}
The controlling of exhaust gas from gasoline is crucial for atmospheric environment protection. Research Octane Number (RON) loss and restricted sulphur (S) content matter the quality of gasoline. To obtain gasoline with high quality, the paper proposes a novel data-driven optimization model integrating deep neural network (DNN) and genetic algorithm (GA) to model for Fluid Catalytic Cracking (FCC) process then optimize. To begin with, the DNN is used to fit the relations between 13 related input variables and output variables in FCC. Subsequently, the FCC process is modelled and GA is proposed to solve the optimization model. Ultimately, 305 samples from real datasets have been analysed to testify feasibility and effectiveness of the method. This paper provides a guideline for the production process of FCC gasoline.
\end{abstract}

Keywords. Exhaust gas, atmospheric environment protection, FCC gasoline

\section{Introduction}

Gasoline is the main fuel for cars, exhaust gas produced by Gasoline combustion has a serious influence on the atmospheric environment. Therefore, countries around the world have formulated increasingly strict quality standards for gasoline, in which the key point of cleaning gasoline is to reduce the content of $\mathrm{S}$ and olefin in gasoline, while maintaining its RON as far as possible. The United States, Britain, Japan and other countries mainly start from the "formulation" to achieve the standard, which is to be blended with gasoline produced through a variety of processes, while the catalytic cracking process is mainly used in China [1]. Because crude oil in China is mainly derived from sour and high-sour crude oil in the Middle East, and it is difficult to directly utilize the medium oil in crude oil. To solve this problem, China has vigorously developed the lightweight technology of heavy oil with FCC as the core to convert heavy oil into gasoline, diesel oil and low carbon olefin. Therefore, more than $95 \%$ of the $\mathrm{S}$ and

\footnotetext{
${ }^{1}$ Corresponding Author, X S Xiao, Bigdata Technology Institute, Guizhou Light Industry Polytechnic College, Guiyang 550025, China; E-mail: shiauxsh@126.com.

${ }^{\dagger}$ Co-first Author
} 
olefins in the finished gasoline come from FCC gasoline. Thus, FCC gasoline must be refined to make the finished gasoline meet the quality requirements of gasoline.

Among the different standards, the most important one is RON, and as the commercial brand of gasoline, it is the most important index to reflect the combustion performance of gasoline. In the process of Desulfurization and olefins reduction of FCC gasoline [2, 3], the RON of gasoline is generally reduced by the existing technology. Each reduction in octane rating by one unit is equivalent to a loss of about $\$ 150$ per ton. If the catalytic cracking process can be modeled, the suitable production variables can be found through simulation experiments so that the RON loss of the finished product gasoline can be decreased on the premise that the quality of the finished product gasoline can meet the environmental protection requirements. Namely, it will reduce the loss of the production cost of gasoline.

Chemical process modeling is generally realized through data-based modeling or mechanism modeling. Mechanism modeling is an accurate mathematical model based on an object, the internal mechanism of a production process, or the transfer mechanism of a material flow [4-8]. In the previous work, Czarnecki et al. [5] presented a model explaining the mechanism of water-in-oil (W/O) emulsion stabilization in petroleum systems. Cao et al. [6,7] investigated the effect of $\mathrm{H} 2 \mathrm{~S}$ in the hydrodesulfurization of FCC gasoline. You et al. [8] predicted the change of FCC based on the dynamic model. Mechanism modeling can infer the model through the existing mathematical formulas, accurately describe the real situation and realize the global optimization, however, due to the complexity of FCC process and the diversity of equipment, considering their control variables are highly nonlinear and strongly coupled. Moreover, the traditional data association model has relatively few variables, and the mechanism modeling has high requirements for the analysis of raw materials, so the response to the process optimization is not timely and performance is not ideal. Subsequently, data-based modeling can make up for these shortcomings. Data-based modeling refers to the abstract organization of all kinds of data in the real world, then determine the scope of the database to be governed, the form of data organization etc. until the database is transformed into a real database [9-11]. Andrade et al. [9] used FT-MIR and PLS methods to predict RON in FCC. Later, Zhou et al. [11] established a prediction model for the increase of propylene in the secondary reaction of FCC gasoline based on L-M algorithm and support vector machine. For complex systems, data modeling is easier to establish a stable model than mechanism modeling because the internal relations of the system are in a black box state. Therefore, this paper adopts a data-based modeling method to improve the FCC process by controlling the main operational variables in the production process. Through numerical simulation experiments, the reliability and stability of the model can be verified, and the input variables can be effectively controlled while the S content is guaranteed. Moreover, the optimization model can be established to minimize RON loss, which can provide effective suggestions and references for reducing RON loss in the real production process.

\section{Model and Method}

In this paper, a two-stage model is established to model and optimize FCC process. Since the chemical system is complex, 13 main features are selected for DNN model. On the basis of DNN, the optimization model is further designed and solved by GA to provide a decision scheme, so as to minimize RON loss on the basis of environment protection. 


\subsection{Deep Learning Model of RON Loss and S Prediction}

The first stage model, as figure 1 shows, the neural network structure for S and RON prediction [12], including the input layer, the hidden layer, and the output layer. The input layer is composed of 13 groups of related independent variables. The hidden layer has been verified by repeated experiments to be the optimal of 7 layers, and each layer includes 16 nodes.

This model is an adaptive neural network, which has introduced dropout method [13], and the probability of dropping nodes is used to transmit information. The white nodes in figure 1 are the discarded nodes. The Dropout method can improve the generalization ability of the model and reduce overfitting. Leaky ReLU is selected as the activation function in this paper. It is not easy to fall into a dead state due to the absence of negative output and has stronger robustness compared with the neural network constructed by traditional ReLU [14]. And adaptive moment estimation (Adam) [15] is introduced as optimization method. It can calculate the adaptive learning rate of different parameters according to the first-order moment estimation and second-order moment estimation of the gradient, and the parameter update is no longer affected by the stretching change of the gradient, which makes model more stable.

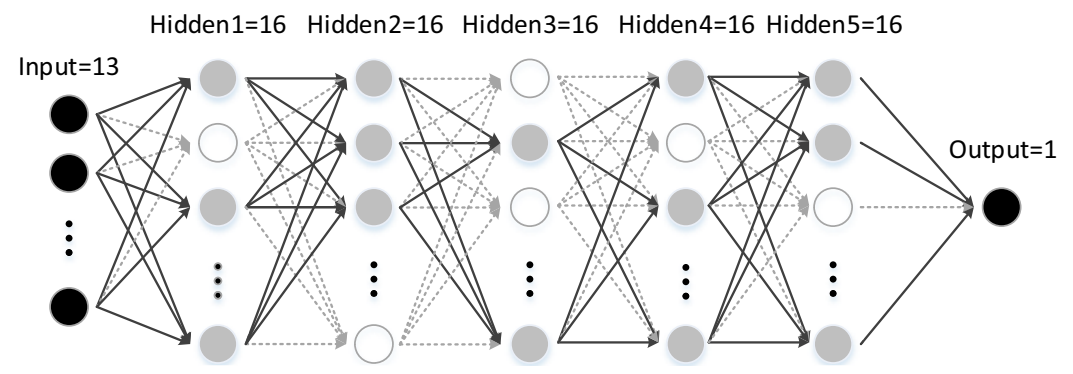

Figure 1. Neural network architecture after dropout.

\subsection{RON Loss Optimization Model Based on Genetic Algorithm}

In the second stage, on the basis of DNN prediction model, RON loss optimization model is established by controlling the main operating variables to optimize RON, so as to reduce RON loss as much as possible in the case of low $\mathrm{S}$ rate. The model is as follows:

$$
\begin{gathered}
x_{1}, x_{2}, x_{3}, x_{4}, x_{5}=\underset{x_{1} \cdots x_{5}}{\arg \min } \operatorname{net}_{1}(X) \\
\text { s.t. } y=\operatorname{net}_{2}(X)<5 \\
a_{i}<x_{i}<b_{i}, \forall i \in[1,5]
\end{gathered}
$$

where $n e t_{1}(X)$ and $n e t_{2}(X)$ are two functions that RON loss and $\mathrm{S}$ content have been trained by DNN in the previous chapter, and $a_{i}$ and $b_{i}$ are respectively the lower bound and the upper bound. 
The constraint conditions and objective function in the optimization model includes the nonlinear function fitted in the previous chapter. Since it cannot be linearized, heuristic algorithm can be used to solve the model. In this paper, genetic algorithm [16] is chosen to solve the model.

Genetic algorithm is a parallel, efficient and heuristic search optimization method based on biological genetics and evolution mechanism. By introducing the biological evolution process of heredity, mutation, selection and elimination into the optimization process. Genetic algorithm has good flexibility and global optimization ability, and provides a general framework for solving complex system optimization problems.

Floating-point coding method [17] is adopted in the genetic algorithm, and the absolute value of the reciprocal of the current RON of the product is taken as the basic value of individual fitness $\mathrm{Jb}$. Considering the limitation of the $\mathrm{S}$ content of the product, the difference between the current $\mathrm{S}$ content value of the individual product and the limit value $(5 \mu \mathrm{g} / \mathrm{g})$ is obtained, and the value obtained after the unit step function filtering (i.e. the negative value of the data is returned to zero) is recorded as the fitness discount coefficient $J$.

Thus, the fitness $J_{i}$ of the $i_{t h}$ individual is expressed as follows

$$
J_{i}=J b_{(i)} \cdot J_{\text {discount }(i)}
$$

The basic value of fitness determines that the core objective of genetic algorithm optimization is to minimize the octane loss (RON). The introduction of the discount factor means that an individual will be eliminated if the $\mathrm{S}$ content of the product exceeds the limit, so that the optimal solution with the greatest reduction in the octane loss (RON) can be found under the strict constraint that the $\mathrm{S}$ content of the product does not exceed the limit.

\section{Simulation and Analysis}

In this paper, the simulation experiment of the model is carried out by Python, and 305 samples are collected from Sinopec Gaoqiao Petrochemical Real-time Database (Honeywell PHD) and LIMS experimental database. There are 13 main features, as shown in table 1 . The first five are operational variables, which can be controlled as independent variables of the model. The others are non-operational variables, which means they aren't decision variables in the second stage model, so they are default to constants in the samples.

\subsection{K-fold Cross Validation for Prediction Model}

K-fold cross validation [18-19], as a verification method of DNN hyperparameters, it obtains as much information as possible from limited learning data, and to a certain extent, avoid the problem of over-fitting and under-fitting. Therefore, we have already taken this method to train the neural network, and test the generalization ability of the trained model, therefore, we can find the most appropriate hyperparameters. 
Table 1. 13 main features.

\begin{tabular}{lllll}
\hline Index & Name & Unit & Min & Max \\
\hline 1 & D-109 pressure & $\mathrm{MPa}$ & 0.1 & 0.15 \\
2 & Nitrogen inlet pressure & $\mathrm{MPa}$ & 0.95 & 1 \\
3 & Stabilize tower top pressure & $\mathrm{MPa}$ & 0.6 & 0.7 \\
4 & D-107 bottom pressure & $\mathrm{MPa}$ & 0.1 & 0.2 \\
5 & Blower inlet pressure & $\mathrm{MPa}$ & -0.1 & 0.1 \\
6 & S content & $\mu \mathrm{g} / \mathrm{g}$ & - & - \\
7 & RON & - & - & - \\
8 & Saturated hydrocarbon & $\mathrm{v} \%$ & - & - \\
9 & aromatic hydrocarbon & $\mathrm{v} \%$ & - & - \\
10 & bromine number & $\mathrm{gBr} / 100 \mathrm{~g}$ & - & - \\
11 & density $\left(20^{\circ} \mathrm{C}\right)$ & $\mathrm{kg} / \mathrm{m}^{3}$ & - & - \\
12 & coke & $\mathrm{wt} \%$ & - & - \\
13 & $\mathrm{~S}$ & $\mathrm{wt} \%$ & - & - \\
\hline
\end{tabular}

This paper conducts nine-fold cross validation for numerical samples. The 305 samples are divided into 9 pieces, of which 8 pieces are used as the training set and 1 piece as the setting of verification, with 100 iterations. Where, the training error train loss represents the loss function value of the training model using the training set data. Namely, the root means square error between the predicted RON loss value and the actual RON loss value. Validation error valid loss represents RON root mean square error obtained by using validation set data after training the model with training set data. As can be seen in figure 2 , the result of each fold and cross validation can be stable after 100 iterations, all the curves are declining continuously. And the loss function can reach the minimum value, indicating that the model is continuously optimized and reaches the optimal value. The calculation results of train loss and valid loss are 0.0758 and 0.785 separately.

Train loss measures the fitting ability of the model on the training set, while Valid loss measures the fitting ability of the model on the new data [20], namely the generalization ability of the model. Both values are very small and close to each other, so our RON prediction model based on deep neural network can be verified to have high accuracy and stability.

In addition, since RON loss and $\mathrm{S}$ content have the same data volume and data characteristics, the same DNN structure is established for S content as that for RON, and the $\mathrm{K}$ fold cross validation is carried out for the prediction model of $\mathrm{S}$ content dataset through simulation experiments. After the same number of iterations, the train loss and valid loss of net 2 are 0.0704 and 0.733 separately, from which it can be seen that the DNN model of $\mathrm{S}$ also has good performance.

\subsection{The Convergence Process of the RON Loss Optimization Model}

As for the parameter setting of the genetic algorithm in the second stage optimization model, after repeated experiments, this paper finally determines that the population size of the genetic algorithm as 50, and the termination evolution algebra is set as 800 generations. When the termination condition is RON lower than $30 \%$, the next generation cannot find a better solution. The crossover probability is 0.5 , and the mutation probability is 0.001 . 

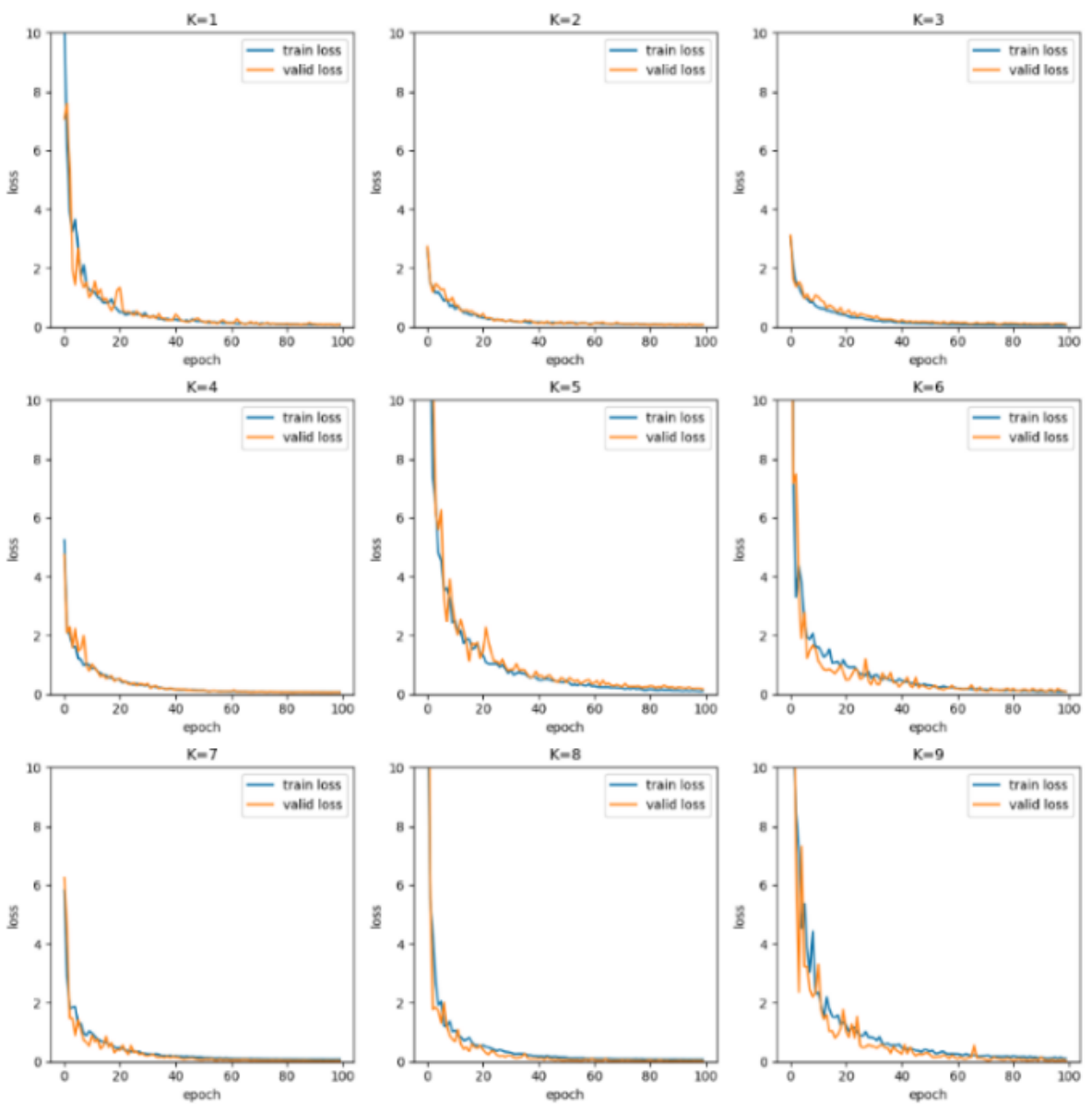

Figure 2. K-fold cross validation result of DNN.

Subsequently, the optimization solution is carried out, firstly we have showed the experiment with sample 1 as an example.

As figure 3 shows, the iterative process of the RON loss optimization model based on genetic algorithm has been shown. Its vertical axis represents the minimum value of individual fitness in the current population. It can be seen that with the increase of iteration times, it gradually decreases to an ideal optimal value in step form. This process well reflects the natural selection characteristics of the genetic algorithm and verifies the effectiveness of the GA.

In this paper, under the premise of ensuring the $S$ content of the product is no more than $5 \mu \mathrm{g} / \mathrm{g}$ to leave the operation space of enterprise (Euro VI and China VI standards are not more than $10 \mu \mathrm{g} / \mathrm{g}$ ), GA is established to optimize all the samples, and the operating conditions of five main operating variables have been obtained to reduce the RON loss of each sample by more than $30 \%$.

Figure 4 shows that the adjustment increment of the five main variables should be made relative to the initial value of their samples. It can be seen as different samples, the adjustment of the operating conditions of the five main variables is mutually influencing 
and competing, which also reflects that it is necessary to apply genetic algorithm of optimizing the RON loss model.

GA is used to solve the optimization model of RON loss and S content, with fast solving speed, accurate solving results and strong model stability. It can provide a clear optimization scheme for cracking gasoline production, and minimize RON loss while ensuring that $\mathrm{S}$ content meets the requirements. Thus industrial chemical production produces the best economic benefit.

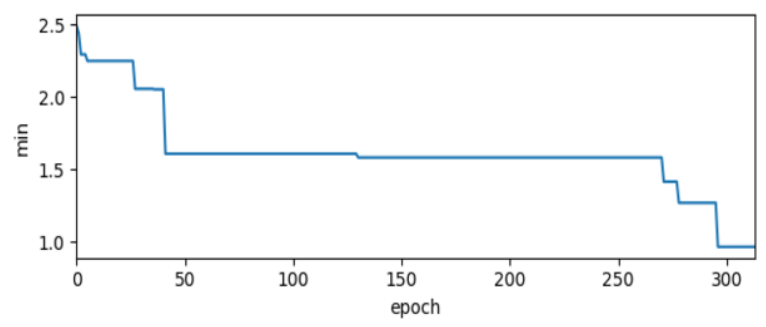

Figure 3. GA convergence process for sample 1.

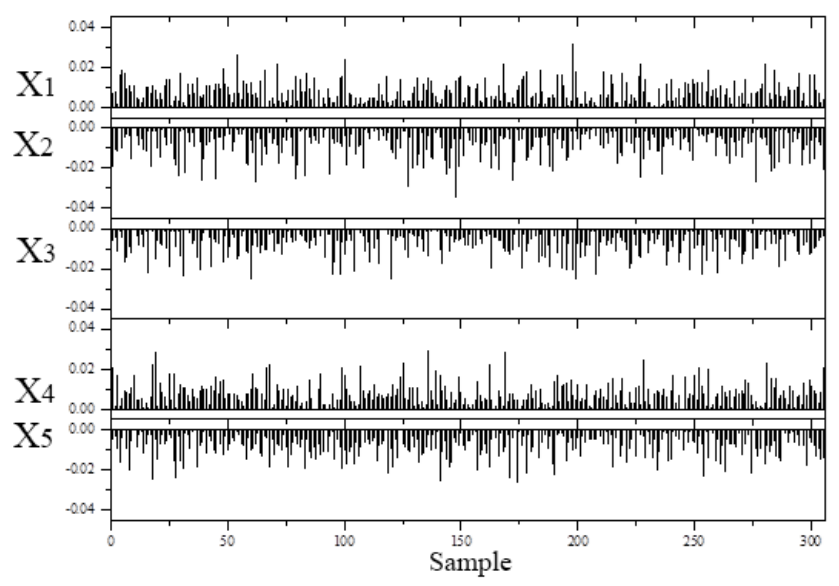

Figure 4. The result of optimization model in all the samples.

\section{Conclusion}

In order to solve the optimization problem of cracking gasoline production in chemical enterprises, a two-stage model has been established in this paper. In the first stage, based on the experimental results, a DNN-RON loss prediction model is set up in this paper. And then the $\mathrm{K}$-fold cross validation test model is adopted to verify the high accuracy and stability of the model. In the second stage, genetic algorithm is adopted to solve the problem, so that the optimal solution with minimum RON loss can be found under the strict constraint of S content of the product. The optimal strategy obtained can provide effective guidance for chemical enterprises to improve efficiency and reduce cost. 


\section{Acknowledgments}

This work is supported by the National Key R\&D Program of China (No. 2019YFB1404901), the National Training Program of Innovation and Entrepreneurship for Undergraduates of China (No. 202010335011) and the Provincial Fundamental Researches Plan of Guizhou (No. [2019]20013).

\section{References}

[1] Xu Y 2014 Advance in China fluid catalytic cracking (FCC) process Scientia Sinica Chimica 44 13-24.

[2] Brunet S, Mey D, Pérot G, Bouchy C and Diehl F 2005 On the hydrodesulfurization of FCC gasoline: a review Applied Catalysis A: General 278 143-72

[3] Jaimes L, Tonetto G M, Ferreira M L and de Lasa H 2008 Desulfurization of FCC gasoline: Novel catalytic processes with zeolites International Journal of Chemical Reactor Engineering 6.

[4] Zeng X L, Liu Y H, Li Y J and Li Y C 2003 Mechanism model for predicting the distributions of wellbore pressure and temperature Journal of Xi'an Petroleum Institute 2.

[5] Czarnecki J and Moran K 2005 On the stabilization mechanism of water-in-oil emulsions in petroleum systems Energy \& Fuels 19 2074-9.

[6] Cao B, Liang Y, Xu C and Gao J 2008 Effects of chemical components on stability of FCC gasoline Petroleum Science and Technology 26 245-55.

[7] Liu B, Chai Y, Li Y, Wang A, Liu Y and Liu C 2014 Kinetic investigation of the effect of H2S in the hydrodesulfurization of FCC gasoline Fuel 123 43-51.

[8] You H 2014 The forecast of nine lumped kinetic models of FCC gasoline under aromatization reaction conditions energy sources Part A: Recovery, Utilization, and Environmental Effects 36 54-63.

[9] Andrade J M, Prada D, Muniategui S and Lopez P 1996 Prediction of FCC gasoline octane numbers using FT-MIR and PLS Fresenius' Journal of Analytical Chemistry 355 723-5.

[10] Wang Z, Yang B, Chen C, Yuan J and Wang L 2007 Modeling and optimization for the secondary reaction of FCC gasoline based on the fuzzy neural network and genetic algorithm Chemical Engineering and Processing: Process Intensification 46 175-80.

[11] Zhou X, Yang B, Yi C, Yuan J and Wang L 2010 Prediction model for increasing propylene from FCC gasoline secondary reactions based on Levenberg-Marquardt algorithm coupled with support vector machines Journal of Chemometrics 24 574-83.

[12] Yousefi M, Yousefi M, Fathi M and Fogliatto F S 2019 Patient visit forecasting in an emergency department using a deep neural network approach Kybernetes 49 2335-48.

[13] Cheng G, Peddinti V, Povey D, Manohar V, Khudanpur S and Yan Y 2017 An exploration of dropout with LSTMs Interspeech 1586-90.

[14] Wang G, Giannakis G B and Chen J 2018 Learning ReLU Networks on linearly separable data: Algorithm, optimality, and generalization IEEE Transactions on Signal Processing 67 2357-2370.

[15] Newey W K 1988 Adaptive estimation of regression models via moment restrictions Journal of Econometrics 38 301-339.

[16] Holland J H 1992 Genetic algorithms Scientific American 267 66-73.

[17] Janikow C Z and Michalewicz Z 1991 An experimental comparison of binary and floating point representations in genetic algorithms Proceedings of the 4th International Conference on Genetic Algorithms 31-6.

[18] Bengio Y and Grandvalet Y 2004 No unbiased estimator of the variance of k-fold cross-validation Journal of machine learning research 5 1089-105.

[19] Fushiki T 2011 Estimation of prediction error by using K-fold cross-validation Statistics and Computing 21 137-46.

[20] Wong T T and Yeh P Y 2019 Reliable accuracy estimates from k-fold cross validation IEEE Transactions on Knowledge and Data Engineering 32 1586-94. 